\title{
Recent applications of Knowledge Organization Systems: introduction to a special issue
}

\author{
Koraljka Golub ${ }^{1} \cdot$ Rudi Schmiede ${ }^{2}$. Douglas Tudhope ${ }^{3}$
}

Published online: 21 November 2018

๑) Springer-Verlag GmbH Germany, part of Springer Nature 2018

\section{Introduction}

Knowledge Organization Systems (KOS), in the form of classification systems, thesauri, lexical databases, ontologies, gazetteers, and taxonomies, more than ever play a crucial role in digital information management and applications generally. Carrying semantics in a well-controlled and documented way, Knowledge Organization Systems serve a variety of important functions such as: tools for representation and indexing of information and documents, knowledge-based support to information searchers, semantic road maps to domains and disciplines, communication tools that provide a conceptual framework, and a conceptual underpinning for knowledge-based systems.

The European NKOS network has held a long-running series of annual workshops at the International Conference on Theory and Practice of Digital Libraries (TPDL), earlier known as the European Conference on Digital Libraries (ECDL). For details of North American and other NKOS network events, see http://nkos.slis.kent.edu/. Special issues on Networked Knowledge Organization Systems (NKOS) have been published in Journal of Digital Information in 2001 (https://journals.tdl.org/jodi/index.php/ jodi/issue/view/8) [1] and 2004 (https://journals.tdl.org/jodi/ index.php/jodi/issue/view/20) [2], the New Review of Hypermedia and Multimedia in 2006 (http://www.tandfonline.

Koraljka Golub

koraljka.golub@1nu.se

Rudi Schmiede

schmiede@ifs.tu-darmstadt.de

Douglas Tudhope

douglas.tudhope@southwales.ac.uk

1 iInstitute, Linnaeus University, 35195 Växjö, Sweden

2 Institut für Soziologie, Technische Universität Darmstadt, Dieburger Straße 203, 64287 Darmstadt, Germany

3 Hypermedia Research Group, Faculty of Computing, Engineering and Science, University of South Wales, Pontypridd CF37 1DL, UK com/toc/tham20/12/1) [3], the International Journal of Digital Libraries in 2016 (https://link.springer.com/journal/799/ 17/1/page/1) [4], and a KOS review article in 2004 [5].

This special NKOS issue of the International Journal of Digital Libraries has evolved from the 16th European Networked Knowledge Organization Systems (NKOS) Workshop at the International Conference on Dublin Core and Metadata Applications 2016 (DC) and the 15th European NKOS Workshop at International Conference on Theory and Practice of Digital Libraries 2016 (TPDL). Following the workshops, a general call for papers was issued. The papers in the issue span broad themes of Linked Data vocabularies, the NKOS community, named entities, automatic indexing, terminological resources, and ontologies (as two examples of KOS).

\section{Special issue papers}

The issue presents five papers on both conceptual aspects and technical implementation of NKOS. These papers are introduced and referenced below.

\subsection{Marcia Lei Zeng and Philipp Mayr: Knowledge Organization Systems (KOS) in the Semantic Web: a multi-dimensional review}

The paper is a state-of-the-art overview of the linked open data (LOD) landscape of Knowledge Organization Systems. Since the Simple Knowledge Organization System (SKOS) specification and its SKOS eXtension for Labels (SKOSXL) became a formal W3C recommendations in 2009, a significant number of conventional KOS (including thesauri, classification schemes, name authorities, and lists of codes and terms) have been transformed to fit into the Semantic Web. This work is a discussion of the impact that the LOD KOS movement has brought to various communities and users. The beneficiaries include not only two traditional groups, i.e., value vocabulary constructors/providers 
and cataloguers/indexers who have a long history of applying the vocabularies to their products, but also LOD dataset producers/LOD service providers, information architects and interface designers, and researchers in sciences and humanities. Based on a set of collected cases, ranging from experimental to implemented applications, the functions of LOD KOS are examined from multiple dimensions.

\subsection{Fariba Karimi, Philipp Mayr and Fakhri Momeni: Analyzing the network structure and gender differences among the members of the Networked Knowledge Organization Systems (NKOS) Community}

The paper provides an overview of NKOS research output of the 16 years in the period from 2000 to 2016, applying a network analytical perspective. To this purpose, an open dataset of bibliographic information has been created that combines 123 publications from European and U.S. NKOS workshops and 4 NKOS special journal issues. The sample comprises in total 256 distinct authors. Three major characteristics are examined: global properties of the network over time, the centrality of the authors in the NKOS network, and gender differences in collaboration behavior. Standard network analytic measures such as degree, betweenness, and closeness centrality are used to describe the co-authorship network of the NKOS dataset. The results show that the authors have higher tendency to collaborate with those in the same institution or within the same geographical proximity. Also, homophily is higher among women in this community. Significant dissimilarities in gender in relation to centralities are absent, apart from a minor degree of differences in closeness and clustering among men and women.

\subsection{Anne-Stine Ruud Husevåg: From subtitles to substantial metadata: examining characteristics of named entities and their role in indexing}

A common topic in the NKOS community is automatic subject indexing. This paper explores the potential of applying named entity extraction from subtitles for automatic indexing of TV programs. The analysis shows that named entities with high frequencies in the subtitles are more likely to be mentioned in the metadata records. Further, the use of named entities in metadata correlates with the frequency of the entities in the subtitles. The named entities with a frequency of two in the subtitles were twice as likely as those with a frequency of one to be present in the metadata records. Personal names, geographical names, and names of organizations were the most prominent entity types in news subtitles and news metadata, while persons, creative works, and locations were the most prominent in culture programs.
The results show that subtitles are a good source for personal names in TV programs.

\subsection{Karolina Suchowolec, Christian Lang and Roman Schneider: An empirically validated, onomasiologically structured, and linguistically motivated online terminology: redesigning scientific resources on German grammar}

The purpose of applying KOS is often that of improved information retrieval. This paper examines onomasiological remodeling of terminological resources for an information system for German grammar called grammis. The major focus is placed on an introduction of features for the authoring backend. A discussion tackles how these innovations help to evaluate existing, loosely structured terminological content, and to efficiently deal with Automatic Term Extraction (ATE). In addition, a transformation of the terminological resource to a future SKOS representation is presented and ensuing benefits for grammis are addressed. The paper is a valuable contribution to the discourse on the place of terminological resources in the world of (Networked) Knowledge Organization Systems.

\subsection{Julaine Sashanie Clunis: Designing an ontology for managing the diets of hypertensive individuals}

Ontologies being a frequently used type of KOS, especially in the context of the Semantic Web, are also a common topic in the NKOS community. This paper describes the development of a specific type of ontology, one which could act as a recommendation system for hypertensive individuals. It puts forward a conceptualization and an implementation of an ontology that describes recipes, food nutrients as well as interactions between nutrients and prescribed drugs, disease, and general health. The ontology model is constructed following the Ontology 101 methodology and validated using competency questions derived from proto-personas. The findings imply that the ontology could be helpful in assisting individuals in navigating complex information spaces when it comes to effects of diet on health. As such, it could be used to provide support to patients seeking to manage chronic illnesses such as hypertension.

Acknowledgements We would like to thank the authors of the contributing papers, all the reviewers who have generously given their time, the various people involved in publishing the issue as well as the participants of NKOS workshops. We hope the articles in the issue will provide a starting point for future explorations in the field. 


\section{References}

1. Hill, L., Koch, T.: Networked Knowledge Organization Systems: introduction to a special issue. J. Digit. Inf. 1(8) (2001). https://journals.tdl.org/jodi/index.php/jodi/article/view/32/ 33. Accessed 19 Nov 2018

2. Tudhope, D., Koch, T.: New applications of Knowledge Organization Systems: introduction to a special issue. J. Digit. Inf. 4(4) (2004). https://journals.tdl.org/jodi/index.php/jodi/article/ view/109/108. Accessed 19 Nov 2018

3. Tudhope, D., Lykke Nielsen, M.: Introduction to Knowledge Organization Systems and services. New Rev. Hypermedia Multimed. 12(1), 3-9 (2006). https://doi.org/10.1080/13614560600856433
4. Mayr, P., Tudhope, D., Dextre Clarke, S., Zeng, M., Lin, X.: Recent applications of Knowledge Organization Systems: introduction to a special issue. Int. J. Digit. Libr. 17(1), 1-4 (2016). https://doi.org/ 10.1007/s00799-015-0167-x

5. Zeng, M.L., Chan, L.M.: Trends and issues in establishing interoperability among Knowledge Organization Systems. J. Am. Soc. Inform. Sci. Technol. 55(5), 377-395 (2004). https://doi.org/10. 1002/asi.10387

Publisher's Note Springer Nature remains neutral with regard to jurisdictional claims in published maps and institutional affiliations. 